\title{
Changing places: Warping between perspectives
}

Alison Gazzard, University of Bedfordshire

\begin{abstract}
The warp device is commonly applied as a design feature within videogames. It allows the player's avatar to be transported through the gameworld changing the relationship with the gamespace. Moving the avatar through the gameworld can be seen as a response to the player's actions and its location acts as a way of working through and understanding relationships within game spaces. This article analyses how warps and portals function and how they can affect the position of the avatar in the gameworld. The space of the videogame is defined through the notion of paths and tracks to understand how players can swap between roles of interaction and observation as they make their way through various levels and scenarios of gameplay. Warps and portals are categorized as to their use within game design in first-person and third-person games and how this impacts on the player's experience of the avatar.
\end{abstract}

\section{Keywords}

Warp portal avatar player position path track 


\section{Introduction}

Videogames often portray fictional spaces. In designing the virtual world of the game, real world rules no longer have to be obeyed. This statement runs true for any fictional design, and it is for this reason that the way space and time are represented in videogames is subject to change. There has always been a fascination with ways of transporting ourselves to other areas through various warp devices, found throughout fiction and more recently, trying to link that fiction with scientific fact. Places such as the rabbit hole in Alice in Wonderland (2007), or objects such as the TARDIS in Doctor Who (1963), and even the bed in Bedknobs and Broomsticks (1971) all act as warp devices, transporting the characters to new places. Each instance of these devices sees the characters within such fictions warp to somewhere new. Through science fiction series such as Star Trek (1966), the term 'warp speed' is also used as a way of describing the way in which the action of warping allows for much quicker movement through space than is generally possible within the real world. This is discussed by Benedikt (in Newman 2004: 11), who states that

the ancient worlds of magic, myth and legend to which cyberspace is heir as well as the modern worlds of fantasy fiction, movies and cartoons are replete with violations of the logic of everyday space and time: disappearance, underworlds, phantoms, warp speed travels, mirrors and doors to alternate worlds, zero gravity [...]. (Benedikt 1991: 128) 
Each instance of the warp acts as an event for changing time, space and narrative elements within fiction. It is through the warp device's presence in various media types that we can recognize it as a common cultural artefact. Therefore, it is only natural that this progression of fiction has been recreated within the videogame, where once again constructions of time, narrative and play can be understood in various ways. Warp devices are a violation of the continuity of the quotidian, and it is accepted that they are not part of our real-world lives. It is through this article that both warp devices and portals are discussed as a way of understanding how the player can move the avatar through the gameworld, and the impact this has on how the player interacts with the avatar in these instances. The terms 'warp' and 'portal' are often used interchangeably within videogame design, yet each has slightly different functions within the videogame. Both the action of the warp device and the portal change the viewpoint of the avatar. However, whereas the warp device visually breaks the player's relationship with the path, the portal offers instant access between two different geographical areas. It is through discussions of the path and the track that the warp and the portal will be further defined within this article.

\section{Defining the warp in videogames}

Super Mario Bros $[\ldots]$ offer(s) warp zones for players to skip levels entirely or to travel down a tunnel to an area without enemies and filled with gold coins to then travel back up a tunnel to the regular level which has been progressed spatially. (Taylor 2006) 
Laurie N. Taylor comments on two types of warp zone found within Super Mario Bros (Nintendo 1985), both found through the use of tunnels. Whereas some tunnels are deliberately installed within the game for players to gain extra rewards such as gold coins or extra lives, other warps can be found through cheats or glitches hidden in the game. Each instance of the warp offers similar yet different experiences and it is these distinctions that will now be explored as an examination of the various characteristics of the warp in videogames. For Jenkins (2006), the 'warp zone' is seen as a place found within the game that is not necessarily intended by the game designer. He writes, “"Secret codes", "Easter eggs" and "Warp Zones" function in digital space like secret paths do in physical space and are eagerly sought by gamers who want to go places and see things others can't find' (in Salen and Zimmerman 2006: 340). Here the warp zone is seen as a distinct type of device, only found by those seeking added extras within the game. However, Jenkins does note how the warp transports the player's avatar from one position to another, and that this may lead to the avatar becoming stuck at their new position in the gameworld.

Various means of transportation between areas within videogames have been discussed using terms such as 'portals', 'teleporters', or 'warp zones'. Rollings and Adams (2003) discuss the use of teleporters within videogames stating that

Teleporters can further complicate matters by not always working the same way, teleporting the player to one place the first time they are used, but to somewhere else the second time, and so on. They can also be one- 
way or two-way, teleporting players somewhere with no way to get back, or allowing them to teleport again. (Rollings and Adams 2003: 231)

Aarseth also discusses the teleporter in terms of how it can move the player's avatar from place to place. He writes, '[a] common motive in many, if not most, computer games is the teleporter, a means to move instantly from one point in the gameworld to another' (Aarseth 2001: 163). Both Aarseth's and Rollings, and Adam's definitions of teleporters can be likened to what this article defines as a 'portal'. It is terms such as portal and warp that will be further distinguished between later on in this article, with the portal allowing for instant movement and the warp device creating a longer warp sequence between places. Aarseth goes on to discuss how this form of instant transportation between places relates back to MUDs (multi-user dungeons/domains) and although MUD administrators tried to keep rooms 'topologically correct', the most common way to move rooms was by using portals rather than moving back and forth through each individual room. Ease of movement in this way was due to players not having a graphical representation of the gameworld, only a text-based description. This type of movement could also confuse the player, forcing them to question their surroundings further and work out new strategies for the area they were now in. Warp zones also allow for a quick escape route in times of trouble, such as the sides of PacMan (Namco 1980) that let the player move the PacMan character from the left side of the screen to the right (or vice versa) in order to avoid the ghosts. Instead of PacMan appearing again instantly, there is a slight delay between entering and existing the warp device. Both warps and portals are a way of extending the space of the gameworld, as a way of making it appear larger than it really is. 
It is through analysing warp zones and devices found in a variety of third-person games, including Super Mario Bros., Ratchet and Clank: Tools of Destruction (Insomniac 2007) and Prince of Persia: Sands of Time (Ubisoft 2003), amongst others, that a set of conditions that define the warp sequence started to evolve. Whereas the presence of warp zones has previously been discussed by others, the actual sequence and action of the warp is often not explored in more detail. Whilst the action of the warp is taking place, the player's relationship with the gameworld is temporarily changed. As each of the descriptions above state, the warp device transports the player from one place to another, therefore changing the player's view of the gameworld as he or she experiences a new event. While the player was once moving his or her avatar along one set of paths within the gameworld, the warp sequence takes him or her to a new set of paths to be explored and discovered. Through breaking the path the player also has a temporary shift in his or her viewpoint of the gameworld and of his or her avatar whilst the warp sequence takes place. For example, when Mario goes down a tunnel in Super Mario Bros., he momentarily disappears, as the gameworld turns black, until he reappears on a new path on the other side. From this it can be seen that the actions of the warp can be defined by the following statements:

- Warp devices break the fluidity of the path. The player is taken from one path to a separate path.

- The player becomes observer for the time they are in the warp sequence, often highlighting the location of the avatar. 
Both of these definitions are linked, as through breaking the path the player temporarily obtains a different relationship with his or her avatar as the route through the gamespace has changed. It is this idea of different routes through the gameworld that will be explored first, which will then lead to a discussion of navigational control and the changing positions of the avatar. Later on in the discussion, it will be shown that the warp can be seen as distinct from the concept of the portal. These once interchangeable terms will be further defined in order to separate their uses within the videogame. Therefore, the notion of the portal and its effect on the gameworld will then be discussed as related to, yet distinctly different from, the warp device in order to discuss the player-avatar relationships further.

\section{Paths and tracks}

It is through the avatar that the player has a representation of his or her progress through space. Moving through the gameworld will be separated into two distinct types of route, that of the path and that of the track. Whereas the path allows for free-form bi-directional movement and spatial exploration of the player's avatar in the gameworld, the track restricts this movement to one direction only, as determined by the game designer. As Gingold (2003) has observed, the locus of the avatar is important for the player to understand where he or she is located within the gameworld. He discusses the 'structure of point of view in participatory media' as being described through four variables including, 'Epistemic Access' understood as 'What can I see? How is the world described to me?' and 'Locus of Manipulation' being understood as 'What do I control, and how do 
I control it?' (Gingold 2003: 82). The locus of the avatar can be linked to understanding the position of the avatar within the gameworld. By combining these notions of Epistemic Access and Locus of Manipulation, we can understand how players make sense of the spatiality of the gameworld through their avatar. The avatar can be understood as a means of being able to guide the player through the space of the game whilst the player is in control. However, moving from paths to tracks can change the avatar's position as well as the interaction available for the player. Therefore, in order to discuss the warp further, routes through space need to be defined.

It is through Aarseth's (1997) discussion of what he terms 'ergodic texts' that the notion of the path in videogames becomes important when discussing spatiality. Although writing in the latter half of the 1990s, when videogames were still predominantly twodimensional for the consumer market, Aarseth's recognition of paths is still relevant to movement through both two-dimensional and three-dimensional videogame spaces. It is through the path that spaces are negotiated. Whereas the spaces, places and architecture experienced by the player may be the same every time the game is played, the paths that the player experiences through this designed architecture may be different. The fixedmaze structure of a game like Pac-Man, for example, remains the same on each play, yet the paths taken in collecting the pills often change each time the maze is negotiated. In three-dimensional games the player cannot always see his or her path before he or she proceeds on it. Paths may change suddenly due to built-in random events, and therefore the situations players encounter on each path lead to the path being unique with most plays even of the same game. This emergent aspect of the path in the display is linked to 
how the player learns to overcome certain paths and unlock others in his or her exploration of the game space. An example of this can be seen clearly in the Playstation 3 game Flower (That Game Company 2009). By using the motion sensing Playstation 3 Sixaxis controller, players control a leaf floating through the gameworld in order to open up closed flowers, creating sounds and unlocking other flowers to open. The player is free to move the leaf in any direction through the bounded space of the gameworld. This definition of the path also extends to side-scrolling games such as Super Mario Bros. Although movement through gamespace is restricted, as the player can never truly go back on him or herself, as he or she moves further to the right of the game the player is still able to move freely along the $x$-axis and $y$-axis that are viewable on the screen for each section of the gameworld. This differs to a game being 'on rails' or consisting of what I define as tracks.

Games discussed as being 'on rails' such as House of the Dead (Wow Entertainment 1997) can be seen as existing on a single track, with occasional branching structures (Fernández-Vara 2007). Due to the goals of the game being more important than any exploratory navigational elements, games such as these exist on tracks rather than paths. Tracks are defined as routes through space that allow for movement in one direction only. Unlike the path, the player cannot move his or her avatar freely along any tracks found within a game. As Nitsche (2008) notes in his discussion of the track metaphor in videogames, 'So-called rail-shooters move or guide the player along invisible tracks that allow little divergence from a given path. The world might appear to be accessible but can be navigated only in the confines of a very limited set track' (Nitsche 2008: 174-5). 
Nitsche discusses the track as an underlying feature on a game path, but if we understand paths and tracks as two types of route through gamespace then the difference between them starts to become clearer. However, tracks are not as straightforward in their definition as paths, and therefore need to be split into further categories for clarity's sake. I define the further divisions as 'tracks with active volition' and 'tracks with suspended volition'. Much like path structures, players do have some element of choice along some tracks in videogames, and it is tracks such as these that allow for active volition. This can be seen in the game House of the Dead. Fernández-Vara (2007) states that

[i]f there is a single path the game feels as though it is 'on rails' like a theme park ride: the user cannot chose where to go. Unicursal structures are this scarce in videogames. Even games that go 'on rails' such as House of the Dead (1997), offer branching paths. (Fernández-Vara 2007: 74)

It is true to say that in House of the Dead, there is no choice as to where the player's avatar can go throughout the majority of the game, making it exist on tracks. However, instead of the paths being seen as branching, these paths are in fact tracks that branch at certain instances within the game. It can be seen that there are multiple tracks to the game, much like different train stations where the player has a choice to get off, and then board another train; the player in this instance is on a track with active volition that is constructed of multiple tracks. The decision of the direction of the track, the speed of track and where the track starts and ends is all taken out of the player's control. However, the tracks allow for some choice from the player's side, such as being able to pause the 
game at a certain moment, and decide whether he or she kills all the enemies in the way of the track or not. Multiple tracks at points within the game levels also allow for some choice as to the direction the player's avatar then goes in, but the tracks restrict general movement such as being able to control movement along the various axes of the gameworld.

In contrast to tracks with active volition, tracks with suspended volition strip away any choice for the player and it is these tracks that can be related to warp devices in videogames. The warp device takes the player's avatar from one position within the gameworld, and in doing so restricts the player's control of the avatar whilst it is in the warp sequence. In many cases, the paths of the gameworld turn into tracks with suspended volition as all navigational control is temporarily stripped from the player until the warp sequence ends. It is segments such as these that are shown through James Newman's discussion about 'ergodicity' in videogames. Newman (2002) states,

[...] videogames do not present a singularly ergodic experience. They are highly structured and comprise episodes of intense ergodic engagement. However, these sequences are punctuated and usually framed by periods of far more limited ergodicity and very often, apparently none at all. (Newman 2002)

The change from the ergodic path to the seemingly non-ergodic track (with suspended volition) within the game highlights the construction of games into sequences of events 
that fluctuate between these two states. It is through this change from path to track through the warp that the player's avatar momentarily becomes the focus of the player's attention. The warp sequence in many ways acts as a shortened cut-scene. The screen of the gameworld may turn to black whilst the player's avatar moves to a new destination, or the game may show swirling visuals, highlighting a warp-speed travel effect between locations. The game controller is temporarily rendered redundant, as the player has to sit and observe what the next game sequence may offer. Therefore, the player's ability to control the location and movement of the avatar is heightened during the warp sequence whilst the player seeks to work out how the warp has worked, where the avatar is now positioned as well as analysing any other events depicted during and after the warp took place.

\section{Changing viewpoints}

In many ways it can be seen that the warp re-positions the avatar. This can be seen in the game Monopoly (Parker Bros. Inc. 1935). If we take the previous definition of warps breaking the fluidity of the path and the player becoming observer whilst in the warp, the game of Monopoly can be seen to have a warp function. Monopoly can be likened to House of the Dead in that it is a game existing primarily on tracks with suspended volition. The player throws a die and can move his or her game piece however many spaces the die depicts. The game piece must be moved in one direction as determined by the game board, but the resultant movement is down to the luck of the die. However, when sent to 'jail' within the game, the player's game piece is taken from the game tracks, making the roll of the die and any resultant movement redundant. By landing on 
the 'Go to Jail' board square or picking up a 'Go to Jail' card, the player's game piece is immediately warped to the jail square of the game board on a track with suspended volition. The rules of the game determine the movement of the playing piece and strengthen this action by stating that the player is not even allowed to collect the $£ 200$ for passing GO, or any other benefits along the way. This is down to the playing piece being transported directly from one square to another and not following the line of the inbetween board squares. The player then has to wait until his or her game piece is freed from jail and able to be moved again along the paths of the game. Whilst in Monopoly jail the player is now forced to take on a different role. For him or her the play of their game has been temporarily halted, and he or she is made to watch the other players' actions until they are free to move their piece back into the space of the gameworld again. The warp in this instance can cause the player to become observer of the gameworld. By observing the action the player is still within the state of play during the game, and acknowledging the wider 'magic circle' (Huizinga 1970) of events. Through observation the player can determine any future moves. Therefore, although interaction is limited, the player's avatar has not left the gamespace. The only change that has occurred is how the gamespace can now be perceived.

The example of warp devices in Super Mario Bros., as discussed previously, also highlights how the player's perception of the avatar is momentarily changed through the warp. Before entering a warp tunnel in the game, the player is able to control the left, right, up and down movements of the Mario avatar. Once in the warp tunnel, Mario disappears, as the player perceives the avatar to be in the tunnel. The screen temporarily 
turns black, emphasizing a change in location. The 'Locus of Manipulation' changes, the player no longer knows what to control or how to control it as the avatar is temporarily removed from the gamespace. It is not until Mario has passed through the tunnel that the player can see Mario again and regain full control. The interaction with the avatar is broken and highlighted by the disappearance of the character on screen. This emphasizes how this track of gameplay is decided by the game designer as a way of moving the avatar in a certain direction. In this instance, the player is often rewarded with a room full of coins that increases the player's game score and detracts the player from thinking he or she is being forced to go a certain way. The tunnel from whence the player's avatar enters the hidden room can no longer be used to exit the room, but the player soon learns that the next tunnel at the end of the room can be used as a warp sequence back out on to the original paths of the game again.

Prince of Persia: Sands of Time is a key example of heightened awareness of the player's avatar whilst it is in the warp sequence. The game contains a special trigger for players to rewind time at certain points within the game, allowing them to stop their avatar falling to its death. As Atkins (2007) notes in his discussion of death within Prince of Persia: Sands of Time, '[b]y spending the accumulated Sands of Time stored in the Sand Tanks of the Dagger the player is able to either move into slow motion, or to reverse time' (Atkins 2007: 243). The capacity to rewind the game indicates a warp sequence within the level for the player. This sequence can be seen as part puzzle and part spatial navigation. It allows the player's avatar, the opportunity to avoid (or delay) death by 
using the player's ability/knowledge of the game to judge when to use the trigger. Whilst triggering the 'Sands of Time Dagger', the game engine controls the player's avatar and interaction with the avatar is temporarily lost. Although the player can still see the avatar on-screen, any control of the avatar is suspended until the rewind sequence comes to an end. The player is only allowed to control the avatar's position in one direction, and does not have full functionality of spatial movement whilst the 'Sands of Time Dagger' is activated. This acts as a warp device, moving the player's avatar from one part of a path back to another along a pre-determined track triggered by the player at that point in the game. Although the player initiates the warp sequence, the resultant action is under the control of the game's system as to how the 'Sands of Time Dagger' warp device functions. The focus of the player's attention whilst the warp sequence is in effect is on the movements of the avatar. Much like being in the Monopoly jail, the player shifts from levels of interaction to observation whilst he or she watches the new position of the avatar take place. This allows for previous pitfalls within the game to be remembered, particularly those that caused for the need of time to be rewound in the first place. The perspective of the player's outlook on the game also changes. Whilst the 'Sands of Time Sand Dagger' is activated, the game screen starts to turn yellowy orange and the graphics warp slightly, showing the previous movements of the avatar in slow motion. At this point the focus is on observing the avatar's movements, and the game controller becomes partially redundant until the sequence ends.

The warp within Prince of Persia: Sands of Time can be defined as a 'return warp' (Gazzard 2009). The player's avatar is returned to the same point on the path it started on 
whilst using the warp function. Return warps, however, are fairly rare to be used purely as puzzles to be solved within games. Whereas the 'Sands of Time Dagger' allows for the player to calculate when to use it in times of need, thus being part of a 'how to save your avatar's life' puzzle to be solved, the majority of return warps tend to be used as a mechanic to make the gameworld appear larger than it actually is. Return warps are generally built into the game system as a way for the player to move his or her avatar between areas more quickly. Therefore, it is often the case that the player's avatar disappears from the screen for a moment, as he or she enters the warp and is re-instated in the gameworld once he or she have come out the other side. Much like the tunnels of Super Mario Bros., the return warp removes player control in favour of a designer control mechanic. The removal of a depicted avatar on-screen once again signifies this momentary loss of interaction. This occurs with the return warp used as a device to transport the player's avatar between levels in Banjo Kazooie: Nuts and Bolts (Rare 2008). The return warp is used in order for the player to move to different areas to play mini-games, and then warp back again to the main town square area where achievements gained are noted and new areas are subsequently unlocked. Whilst the avatar disappears whilst in the warp sequence, the screen of the game produces a 'warping graphic', where swirling colours are shown as a way of depicting that time and location have changed. Although the avatar has disappeared, the player's action is still taken from paths to tracks. The interaction with the gameworld is once again halted whilst the graphics take place and only resume once the avatar returns to the screen ready to be controlled once more. This theme is common in 'jump warps' (Gazzard 2009) where the avatar jumps from one path of the game to another. Unlike return warps, the jump warp takes the player's avatar 
somewhere new in the gameworld each time. Again there is a tendency for the avatar to temporarily disappear on-screen, highlighting loss of interaction for the player until it returns in a new place within the game. The temporary disappearance of the avatar extends the space of the gameworld, making locations feel further apart than if the avatar had moved along a path to get there. The spatial navigation is then determined by the designed track of the gameworld as opposed to the free-form path. This is shown in Ratchet and Clank: Tools of Destruction, where jump-warp platforms are deliberately installed within the level design. The jump warps restrict the movement of the avatar and allow the player to observe more of the gameworld whilst interaction is on pause. Any path navigation is restricted in favour of a pre-determined track that takes the avatar across the gameworld. This also changes the time of the gameplay. The speed of the avatar's movement is increased during these warp sequences as spatial navigation is minimized. The gameworld appears larger as, instead of the player moving the avatar through on a path-by-path basis, the player as observer views the gameworld from a different perspective. Although the player may not be able to move through some of the architecture shown from the warped view, the experience fills in extra details of the games landscape allowing it to feel much larger and more explorable than it actually is.

If warp devices change the state of interaction for the player shifting the emphasis from player as controller to player as observer, then the case of the cut-scene needs to be investigated further to understand whether it too can be defined as a warp. Juul (2005), Crogan (2003) and Newman (2002) have all written about the use of the cut-scene within videogames, how they can restructure time and fill in narrative sequences for players in- 
between more ludic, player-driven events. In many level based games, cut-scenes can denote the completion of one level and the beginning of a new one. In this case the previous levels' path is broken, causing these cut-scenes to act as level-warps. Unlike the warps discussed previously, the player does not trigger the warp device in these instances. Instead, the level-warp is triggered by the player completing one level and the game system automatically transporting him or her to the next playable level. The old path is now left behind, and the player's avatar is warped to a new level faced with a new set of paths to overcome and understand after the cut-scene has taken place. The cutscene in this respect can be seen as a level-based, jump warp moving the gameplay in one direction. The player has no control over where his or her avatar is taken to after the cutscene, the paths of the game are broken and new paths have to be learned and experiences built upon from previous paths encountered in the game.

Breaking the path and playing a pre-rendered or in-game movie also changes the player's relationship with how he or she can interact with their avatar. This is particularly evident in the cut-scenes included in Heavy Rain (Quantic Dream 2010) or Grand Theft Auto IV (Rockstar Games 2008). Each time a new section of the game has been completed, the interaction stops and the action is put on tracks as determined by the next narrative cutscene sequence that ensues. Cut-scenes share common features with warps that have been discussed previously. As well as suspending the interaction, the avatar-path relationship is also changed. Instead of the player having control over where the avatar moves along the path, the path turns into a track decided by the game designer in creating the cutscene animation. The player is no longer linked to the avatar through actions inputted via 
the game controller, and he or she are made to observe the following action in order to understand the next sequence of events. The position of the avatar also changes. In Heavy Rain, each cut-scene is shown through a close-up of the face of the next character you will play as. Any previous interaction with the in-game camera or movement of the avatar is now determined by the programmed elements within the game engine. This happens in many first-person and third-person game cut-scenes, where the avatar is repositioned on the screen. In cases where the player often 'follows' the avatar by controlling them from behind, the face and front view of the avatar then shift into focus during the cut-scene while facial expressions and body language become more prominent in portraying what is occurring during these linked narratives.

It is through examples such as these that some cut-scene events can be discussed as levelbased warps, taking the player from one part of the gameworld to another, bridged by the narrative of the temporary warp. However, not all cut-scenes by definition are warps. Some games such as Left 4 Dead (Certain Affinity 2008) and the opening sequence to the game Half-Life (Valve Corporation 1998) allow players to control certain levels of interaction with his or her avatar, in that, during a scene where the game is still loading, the avatar can be moved in a limited environment. Here, the avatar is placed on tracks with active volition, as navigation is controlled yet it is not wholly pre-determined. Therefore, some cut-scenes act as a way of bridging the narrative or allowing a game to load more data through keeping the avatar on the same path, whereas others allow for the game to be taken to a new place or level, therefore warping the avatar from its original path and on to a new one. However, some cut-scenes act as part of the construction of 
puzzle warps in games. They are moments to be sought out in order to progress, and jumping from level to level retains this momentum for the player as he or she continues to work their way through the game.

\section{The portal versus the warp}

It is through these discussions of the warp device and its ability to break the path in videogames that the issue of time also becomes relevant. Through changing the potential to interact with the avatar, the warp temporarily puts the avatar on pre-designed tracks rather than player-defined paths. The amount of time for which the avatar moves along these tracks can also be changed in order to speed up the warp sequence, as was seen in the discussion of the jump warps of Ratchet and Clank: Tools of Destruction. In returning to the initial discussion of warps, it can be seen that time is a related factor in defining the portal. Whereas the portal could be seen as a warp device in that it can temporarily change the player's relationship with the avatar, the portal breaks spatial progression instantly; therefore the avatar is never put on tracks. As stated within the introduction, the portal allows for instant movement between different spaces within the game. Although the speed at which the avatar can move between different rooms does start to change, interaction with the avatar remains intact whilst it moves through the portal. Unlike the warp, the portal does not put the avatar on tracks but allows for instant movement, and a one-to-one relationship between the player's control and the avatar's movement remains. However, the portal continues to break the original path as the player is transported from one geographic area in the game to another. The portal allows the properties of otherwise 
impenetrable objects to be temporarily changed enabling movement between seemingly unconnected areas.

On a first examination, it can be seen that the game Portal (Valve Corporation 2007) contains jump warps as determined by the player's positioning of the warp. However, Portal offers a unique experience that breaks some of the conditions usually associated with warp devices such as placing the avatar's action on tracks within the gameworld. It is through noticing the differences between warps and portals that the two terms can be separated. At the beginning of Portal the player learns how the portal works, allowing the avatar to move from one path to another by using a pre-determined start point to the warp, and positioning his or her own end point (Gazzard 2009). The game is based around solving puzzles through placing the portal in different areas of the room in order to progress to the next level. At first the player is only able to create the end point to the portal, but is soon given the freedom to position both start and end points in order to reach his or her goal. Portals have to be placed in the correct position in order to solve puzzles along different paths by making the paths safe before the player's avatar moves to the new path and carries on its journey. Although it is a game based around breaking the path of one place in order to warp to a new path to solve the puzzle, the momentum of moving through the warps means that the warp does not force the avatar on to tracks whilst they are warping. In this instance, the portal warps the player's notion of the gamespace but does not change temporal or interactive elements as the player remains in control of the avatar at all times. Moving through the portal is instant, allowing the player to continually keep moving the avatar or various objects through each room. Once 
through the portal, the door closes and a new portal has to be created. Therefore, the original path is distinctly broken each time, as the player's avatar moves to a new geographic space. However, the portal breaks the convention of the navigational control being removed such as in the other warps discussed previously. If the avatar is positioned right at the centre of the warp, the game system does try to force it either in or out, but this is a rare case, as most players will try to move their avatar straight through the portal without stopping. Navigational control is not removed through using a portal, but understanding the player's relationship with his or her avatar through the space of the game becomes part of the portal's puzzle to be solved.

Portal is played from what is commonly termed a first-person perspective. The player has a viewpoint of the avatar's portal weapon but all other parts of the body are not in view. The creation of the portal warp within the game changes this perspective. By moving the avatar near the portal warp at certain points, the whole of the avatar becomes displayed in the portal that it will be warped to. This changes the player's viewing relationship with the avatar, with the avatar being viewed in both first-person and third-person at the same time. The relationship shifts from the player being able to interact with the avatar, to only being able to observe its actions as the player continues to reposition the portal to understand its relationship with the space it is revealing. The 'locus of manipulation' as a way of understanding the player's connection to the avatar he or she is moving through space starts to change through the positioning of the portal. Although the player is still linked to the avatar, the distortion of space allows for the avatar to be viewed as it interacts with and moves through a seemingly new space of the gameworld. This is part 
of the challenge of Portal in working out where the avatar is in space, therefore the change in perspective allows for confusion as to where the avatar is located in the gameworld in relation to other events. Although the avatar is not placed on tracks whilst moving through the portal, the associated characteristics of tracks in relation to observing the avatar is still possible through the placement of start and end points to the warp. Whereas in previous warps, where the avatar disappears or is shown in another position and then returns to its normal state outside of the warp, the portal allows for a combination of these events to occur simultaneously. This once again reinforces how warps and portals can heighten the avatar's location, and related player interaction linked to whether the avatar can be repositioned by the player or by the game's system (or both).

\section{Conclusions}

The above discussion of different types of warp shows how warp devices change the position of the avatar in the game, heightening the player's awareness of the character and often limiting the avatar's subsequent position at the end of the warp sequence. In contrast portals allow for continuous movement, therefore loss of interaction does not occur, yet the new position of the avatar may then cause the player to have to re-negotiate new paths or try and make their way back to old ones. The warp device shows how games are not always continuously ergodic, echoing Newman's discussion of the 'Myth of the Ergodic Videogame' (Newman 2002). By understanding routes through space it is possible to see how players can move from game paths to game tracks with various degrees of interaction and observation in between. Even in moments of limited interaction, the warp allows for the player to remain within the overall space of gameplay 
through observing past and future events and anticipating their next move. Warps and portals are also used as inbuilt puzzles in order to distort the space of the game and the player's relationship with their avatar.

Understanding the position of the avatar in relating how the player's actions are translated into the game world (through the 'Locus of Manipulation') highlights how the warp can disrupt these actions. Finding warps hidden within levels, such as the glitches found at the end of Level 1-2 of Super Mario Bros., can transport the Mario avatar to a level that can be played through, yet offers no further progression at the end of it. In this case the player is rendered in continuous interaction or a continuous observation of the environment until the level is reloaded and the intended game is restarted. Each deliberately installed warp found within a game world usually seeks to extend the space of the game, making it appear larger than it actually is in terms of temporal and spatial exploration. This is also true of cut-scenes that can offer level-progression warps and further extend the narrative elements of the gameworld in shorter, more direct sequences compared to unfolding the narrative through spatial exploration. Warps and portals seek to progress the player in one way or another, and can be seen to extend the time the game is played for. Therefore, it can be seen that until technology allows for infinitely expandable game spaces, warp and portal devices are key elements in setting up sequences of interaction and observation as players seek to negotiate new, explorable game content.

\section{References}


Aarseth, E. (1997), Cybertext: Perspectives on Ergodic Literature, Baltimore, MD: Johns Hopkins University Press.

(2001), 'Allegories of Space: The Question of Spatiality in Computer Games', in M. Eskelinen and R. Koskimaa (eds), Cybertext Yearbook 2000, Saarijarvi: Gummerus Printing, pp. 152-71.

Atkins, B. (2007), 'Time in Prince of Persia: Sands of Time', in B. Atkins and T. Krzywinska (eds), Videogame, Player, Text, Manchester: Manchester University Press, pp. 237-53.

Benedikt, M. (1991), 'Cyberspace: Some Proposals', in M. Benedikt (ed.), Cyberspace: First Steps, Cambridge, MA: MIT Press, pp. 119-224.

Carroll, L. (2007), Alice's Adventures in Wonderland, London: Penguin Classics.

Certain Affinity (2008), Left 4 Dead, Bellevue, WA: Valve.

Crogan, P. (2003), 'Gametime: History, Narrative and Temporarily in Combat Flight Simulator 2', in M. J. P. Wolf and B. Perron (eds), The Video Game Theory Reader, London: Routledge, pp. 275-302. 
Fernández-Vara, C. (2007), 'Labyrinth and Maze', in F. von Borries, S. P. Walz and M. Böttger (eds), Space Time Play, Computer Games, Architecture and Urbanism: The Next Level, Basel: Birkhauser, pp. 74-87.

Gazzard, A. (2009), 'Tunnels, Teleporters and Time: Warp Devices in Videogames', in DiGRA2009: Breaking New Ground, Brunel University, 1-4 September, Uxbridge, London: United Kingdom.

Gingold, C. (2003), 'Miniature gardens, and magic crayons: game, space and world', Masters Thesis, Atlanta, Georgia: Georgia Institute of Technology.

Huizinga, J. (1970), Homo Ludens: A Study of the Play Element in Culture, New York: J \& J Harper Editions.

Insomniac (2007), Ratchet and Clank: Tools of Destruction, Foster City, CA: Sony Computer Entertainment.

Jenkins, H. (2006), 'Complete Freedom of Movement: Videogames as Gendered Play Spaces', in K. Salen and E. Zimmerman (eds), The Game Design Reader, Massachusetts: MIT Press, pp. 330-65.

Juul, J. (2005), Half-Real: Video Games Between Real Rules and Fictional Worlds, Massachusetts: MIT Press. 
Namco (1980), PacMan, Tokyo: Namco.

Newman, J. (2002), 'The myth of the ergodic videogame', Game Studies, 1:2, http://gamestudies.org/0102/newman/. Accessed 17 June 2010.

(2004), Videogames, London: Routledge.

Newman, S., Webber, C. E. and Wilson, D. (1963), Doctor Who, UK: BBC.

Nintendo (1985), Super Mario Bros, Osaka: Nintendo.

Nitsche, M. (2008), Video Game Spaces, Cambridge, MA: MIT Press.

Parker Bros. Inc. (1935), Monopoly, England: Waddingtons.

Quantic Dream (2010), Heavy Rain, Foster City, CA: Sony Computer Entertainment.

Rare (2008), Banjo Kazooie: Nuts and Bolts, Redmond, WA: Microsoft Game Studios.

Rockstar Games (2008), Grand Theft Auto IV, Edinburgh: Rockstar North. 
Roddenberry, G. (1966), Star Trek, Culver City, CA: Desilu Productions.

Rollings, A. and Adams, E. (2003), Andrew Rollings and Ernest Adams on Game Design, Indianapolis, IN: New Riders.

Stevenson, R. (1971), Bedknobs and Broomsticks, Dorset, UK: Walt Disney Productions.

Taylor, L. N. (2006), 'Labyrinths, mazes, gardens, and sandboxes: game space metaphors', in GameSetandMatch II: On Computer Games, Advanced Geometries, and Digital Technologies (Proceedings), Delft University of Technology, The Netherlands, 29 March-1 April 2006, Rotterdam, Netherlands: Episode Publishers, pp. $98-106$.

That Game Company (2009), Flower, Foster City, CA: Sony Computer Entertainment.

Ubisoft (2003), Prince of Persia: Sands of Time, Montreal: Ubisoft Montreal.

Valve Corporation (1998), Half-Life, Kirkland, WA: Sierra Studios. (2007), Portal, Bellevue, WA: Valve Corporation. 
Wow Entertainment (1997), House of the Dead, Tokyo: Sega.

\section{Contributor details:}

Alison Gazzard is a Post-Doctoral Research Fellow in New Media at the University of Bedfordshire, UK. She holds a Ph.D. from the University of Hertfordshire titled 'Paths, Players, Places: Towards an Understanding of Mazes and Spaces in Videogames' and MA in 3D Computer Animation from the National Centre of Computer Animation at Bournemouth University. Her research has been presented internationally at conferences such as ISEA2008, ISEA2009 and DiGRA 2009. Her current research interests include paths, journeys and time in gamespaces, location-based media, augmented reality games, and understanding player-avatar relationships in virtual worlds.

\section{Contact:}

Research Institute for Media, Art and Design, University of Bedfordshire, Park Square, Luton, LU1 3JU, UK.

e-mail: agazzard@me.com. 